\title{
Application des données de l'Inventaire Forestier National à l'étude de la production du pin sylvestre en Margeride
}

\section{B. - Etude de la production totale en volume}

\author{
J.M. OTTORINI \\ I.N.R.A., Station de Sylviculture et de Production \\ Centre de Recherches forestières de Nancy, \\ Champenoux, F 54280 Seichamps
}

\begin{abstract}
Résumé
Dans la seconde partie d'une étude consacrée à l'estimation de la production totale en volume du pin sylvestre en Margeride, des relations entre les caractéristiques des placettes utilisées ont été recherchées pour obtenir une expression du volume sur pied et de l'accroissement courant en volume des placettes qui pouvait convenir aux besoins de l'étude. Une relation liant l'accroissement courant en volume à l'âge, la hauteur et la surface terrière a ainsi été mise en évidence. En s'appuyant sur les courbes de croissance en hauteur issues de la première partie de l'étude, et sur une relation donnant la surface terrière des peuplements sur pied en fonction de la hauteur, l'expression de l'accroissement courant en volume a pu être intégrée en fonction de l'âge pour des classes de productivité données, conduisant ainsi à la production totale. De plus, pour avoir une meilleure idée du développement des peuplements, le nombre de tiges à l'hectare correspondant généralement à un âge, une hauteur, et une surface terrière donnée a pu être obtenue.
\end{abstract}

\section{1. - Introduction}

Cet article présente les résultats finaux d'une étude basée sur les données de linventaire Forestier National et ayant eu pour but de déterminer la production des peuplements de pins sylvestres dans la région de la Margeride. Les motivations. des généralités, et les premiers développements de cette étude ont été exposés dans une publication déjà parue (OTTORINI \& NYs, 1980). Les travaux présentés ici s'appuient sur ces premiers développements; il s’imposait de distinguer deux parties dans cet ensemble pour pouvoir traiter de problèmes de natures très différentes.

Une difficulté majeure dans l'étude de la production forestière tient au fait qu'à chaque moment le volume sur pied d’un peuplement n'est qu'une fraction du volume produit depuis son origine par les arbres dont il se composait alors; c'est 
cette quantité habituellement appelée "production totale» quiil est véritablement important de connaître, et les moyens de la déterminer dépendent des données disponibles. Si l’on dispose de placettes qui ont été régulièrement suivies et mesurées depuis que leur peuplement existe, la production totale peut en être connue directement, mais cette situation est exceptionnelle. Aussi, Iorsque l'on doit s'appuyer sur des placettes qui n'ont été mesurées qu'une seule fois comme ce fut le cas ici (placettes dites temporaires), ou sur des placettes qui n'ont été mesurées de façon suivie que pendant un temps relativement court, pour en déduire la production totale à l'unité de surface de l'essence considérée il faut avoir recours à des artifices. Ces moyens artificiels reposent à la fois sur des faits que les mesures permettent de mettre en évidence et sur d'indispensables hypothèses (basées sur des connaissances générales antérieures) venant suppléer au manque d'informations. La section suivante détaille dans ce cadre les principes et les méthodes qui ont permis de parvenir à une solution du problème initialement posé.

\section{2. - Principes et méthodes}

\section{1. - Principes dendrométriques généraux}

Lorsque la production totale des peuplements forestiers n'est pas directement observable, c'est-à-dire lorsque l'on ne dispose pour la déterminer que de placettes temporaires ou semi-permanentes, il faut la calculer en exploitant les relations qui peuvent apparaître entre les diverses composantes de la croissance des peuplements à travers les mesures dendrométriques dont on dispose. Dans tous les cas, faire ce calcul signifie que l'on a pu obtenir une expression liant l'accroissement en volume des placettes aux autres caractéristiques de celles-ci dont l'évolution est plus facile à connaître. Par exemple, dans la méthode utilisée en France pour la construction de tables de production à partir de placettes temporaires (DÉcourT, 1972) le calcul de la production totale est basé sur le rapport $\mathrm{K}$ liant le volume enlevé en éclaircie au volume sur pied : si l'on néglige la mortalité naturelle entre éclaircies, l'évolution du rapport $\mathbf{K}$ et du volume sur pied déterminent l'accroissement courant. Cette méthode ne nécessite pas de sondage à la tarière mais s'applique seulement aux peuplements assez régulièrement éclaircis. En ce qui concerne le pin sylvestre en Margeride dont il semble que la sylviculture soit pratiquement inexistante en général (voir Marquestaut, 1979) il fallait employer d'autres moyens pour en étudier la production. Les données de l'I.F.N. incluant des mesures d'accroissement de la grosseur des tiges effectuées à la tarière à $1,30 \mathrm{~m}$, il était possible de rechercher en se basant sur ces mesures - une relation déterminant la production totale à partir de l'accroissement courant : cette relation devait être donc de nature différentielle et son intégration pouvait conduire à la solution. Il semble que l'on puisse attribuer la première tentative de ce genre à Buckman (1962), et les travaux de ClutTer (1963) \& Curtis (1967) marquent d'importantes étapes dans la réalisation d'un tel programme. Les démarches qui apparaissent dans ces articles sont cependant toutes distinctes, elles dépendent à la fois des circonstances particulières à chaque étude et de l'importance que chaque auteur a attaché à tel ou tel aspect des choses; aussi pour ces mêmes raisons la démarche présentée ici se distingue des précédentes par 
plusieurs points. Enfin pour clore cette discussion, il reste à dire que les schémas simplificateurs mettant en évidence lindépendance de certains phénomènes ou l'invariance de certaines relations sont des plus précieux (voir DécourT, 1972). Il est absolument nécessaire de rechercher des possibilités de simplifications (exactes ou approchées) pour étudier la croissance et le développement des peuplements forestiers dont l'état est déterminé par un grand nombre de variables qui sont toutes plus ou moins liées. En particulier, bien que cette hypothèse n'était pas vérifiable dans le cadre de cette étude, mais comme cela est généralement admis, dans les développements qui suivent la hauteur des peuplements - ici la hauteur moyenne des arbres dominants et codominants, conformément à ce qui a été convenu dans la première partie de l'article — qui est un élément central de référence, est supposée dépendre uniquement de la productivité du lieu et de lâge des peuplements (ce qui en général n'est jamais gravement contredit par les faits expérimentaux).

\section{2. - Méthodes d'analyse des données}

Dans le développement des prochaines sections apparaîtront des relations faisant intervenir simultanément plusieurs caractéristiques de peuplements liées par diverses fonctions ; quelques explications sont nécessaires à ce sujet, en particulier pour montrer comment la forme de ces relations a pu être établie avec sûreté. Des connaissances générales préalables facilitent la recherche de relations entre les caractéristiques dendrométriques d'un échantillon de peuplements forestiers, mais cette recherche n'est vraiment efficace que si elle est conduite méthodiquement. Pour étudier la dépendance de deux variables il n'y a pas de substitut aux graphiques où l'on observe dans un système de deux axes les points ayant pour abscisses et ordonnées les variables à étudier. Ces graphiques (ou diagrammes de dispersion comme ils sont encore nommés) ont des rôles complémentaires : ils rendent apparente une relation entre deux variables si elle existe, ils indiquent la forme fonctionnelle de cette relation abstraction faite d'une certaine variabilité, et ils indiquent l'importance de cette variabilité inexpliquée. Une hypothèse faite sur la forme fonctionnelle de la relation peut être alors facilement vérifiée : lorsque la transformation correspondante est appliquée à l'une des deux variables (ou aux deux), alors les points doivent se disperser autour d'une droite; si ce n'est pas le cas l'hypothèse peut être révisée. Enfin, quand une dépendance a été détectée entre deux variables, l'analyse décrite peut être poussée plus loin aussi complètement avec la même simplicité pour étudier la possibilité que l'une de ces variables soit en fait simultanément dépendante de deux autres : pour cela les opérations déjà effectuées constituent une étape qui permet d'estimer une relation provisoire, le pas suivant consiste à étudier avec les mêmes techniques la dépendance des valeurs résiduelles issues de cette estimation vis-à-vis de nouvelles variables (les valeurs résiduelles sont les différences entre les valeurs observées d'une variable convenue «dépendante » et les valeurs estimées par une fonction de la variable «explicative»; on peut se reporter à Draper \& Smith, 1966, CH IV; Mc Neil, 1977, CH III; ou Mosteller \& Tukey, 1977, p. 271, pour plus de détails sur l'ensemble de ces questions).

Lorsque la forme définitive d'une relation est connue il reste à procéder à l'estimation des paramètres qui doivent y figurer. Comme l'estimation des formes linéaires est accomplie par les méthodes les plus simples à mettre en œuvre et qui donnent les résultats les plus satisfaisants, il est préférable d'y avoir recours chaque 
fois que cela est possible. Quand ces circonstances favorables ne se présentent pas directement, la transformation d'une ou de plusieurs variables évoquée au paragraphe précédent permet très souvent de s'y ramener, mais dans chaque cas particulier peuvent se présenter d'autres possibilités qui permettent de le faire : il se trouve que chaque fois au cours de l'étude qui a donné lieu aux développements qui suivent le problème d'ajustement qui se posait a pu être ramené à celui d'un ajustement linéaire. Pour ces estimations linéaires la méthode des moindres carrés est préférable aux autres dans les conditions où on peut l'appliquer. En d'autres circonstances elle doit cependant être évitée (ou modifiée) en particulier lorsqu'il n'y a pas homogénéité de la dispersion des valeurs observées au voisinage des valeurs à estimer. Dans ce cas des méthodes basées sur un principe différent (dans lesquelles la médiane joue un rôle important) ou dérivées (emploi de pondérations) peuvent être substituées à la méthode des moindres carrés (voir Mc NeIL, 1977, p. 49 ; MostelLer \& Tukey, 1977, p. 356, pour plus de détails). Par deux fois le cas s'est présenté, et pour cela le traditionnel coefficient de corrélation n'accompagne pas les relations déterminées, mais les graphiques correspondants peuvent suggérer avec quelle précision elles sont vérifiées (plus précisément, l'estimation des paramètres qui apparaissent dans les relations (2) et (5) a nécessité l'emploi de pondérations calculées de façon itérative dans le cadre de la méthode des moindres carrés; les résultats obtenus ont été contrôlés, comme dans tous les autres cas, par un examen fínal des résidus conjointement avec les, valeurs estimées).

\section{3. - Relations conduisant à une expression de la production totale}

Dans cette section sont présentées deux relations fondamentales que les données étudiées ont permis d'établir : la première liant le volume sur pied d'une placette à la surface terrière et à la hauteur est bien connue, la seconde liant l'accroissement relatif de la surface terrière sur pied à l'âge ne semble pas avoir été déjà signalée. L'application de ces relations est ensuite envisagée.

Le volume de chaque arbre pris en compte sur une placette de l'I.F.N. est directement évalué à partir des mesures de deux billons, le premier qui va du pied de l'arbre jusqu'à $2,60 \mathrm{~m}$ avec la mesure du diamètre à $1,30 \mathrm{~m}$, le second qui va de $2,60 \mathrm{~m}$ jusqu'à la hauteur de la découpe bois fort avec la mesure du diamètre médian ; à chaque placette est donc finalement attribué un volume total $\mathrm{V}$ provenant de ces mesures. Une relation entre le volume total des placettes, leur hauteur $\mathrm{H}$ et leur surface terrière $G$ est apparue être vérifiée avec une très bonne précision (voir la figure 1) et estimée comme il suit:

$$
\frac{V}{G}=0,21303 H^{1,2}+1,3739 \quad R^{2}=0,94(268 \text { observations })
$$

(V en mètres cube par hectare, $G$ en mètres carré par hectare, $H$ en mètres. $C e$ résultat est à rapprocher de la classique relation $V / G=F H$ où $F$ est le « coefficient de forme », qui a eu cours pour d'autres auteurs). 


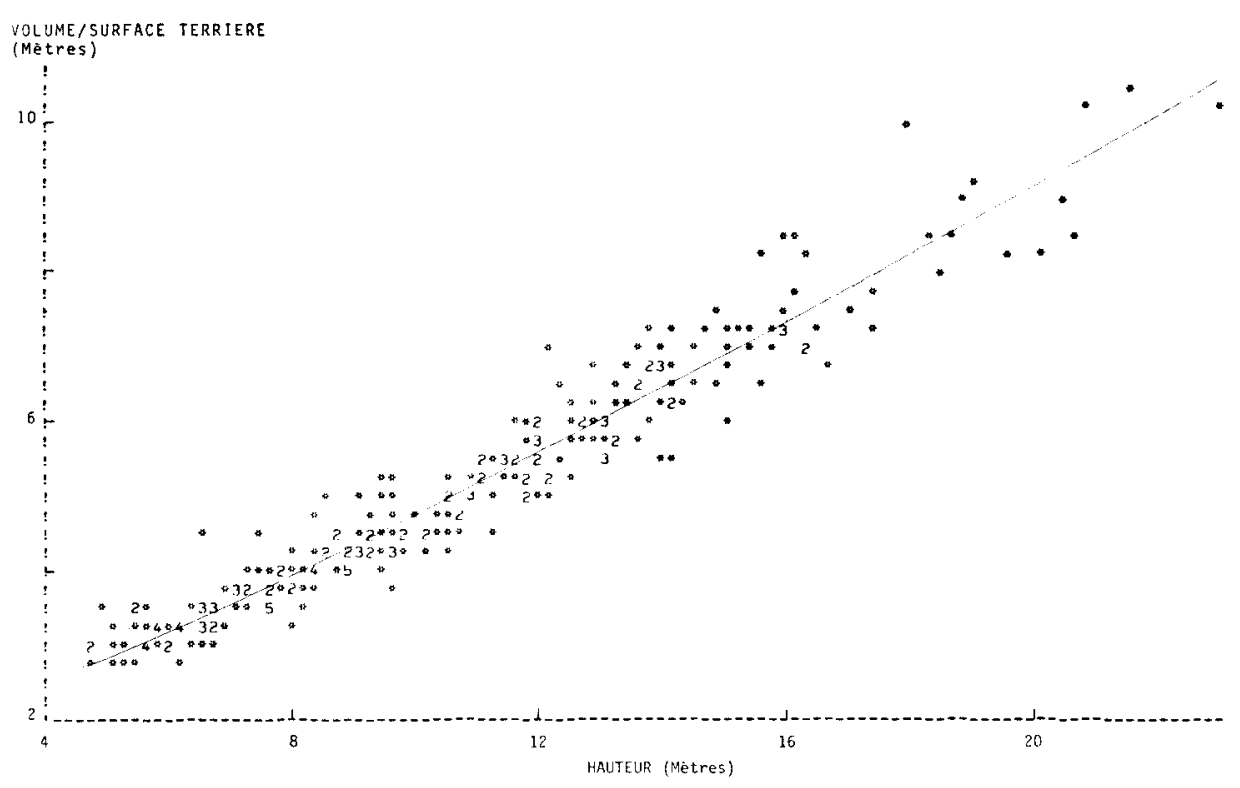

FIG. 1

Relation entre la hauteur et le rapport du volume à la surface terrière des placettes de l'étude. Comme dans les figures suivantes, toutes les caractéristiques de peuplement sont exprimées à l'hectare (ceci n'est pas précisé sur les axes)

Relationship between height and ratio of volume to basal area of the sampled stands (values of stand characteristics are for one hectare; height is the mean height of the dominant and co-dominant trees, and practically may be confounded with the mean height)

Par ailleurs, les mesures individuelles des 5 derniers cernes d'accroissement annuel en circonférence à $1,30 \mathrm{~m}$ effectuées à la tarière, et de l'épaisseur d'écorce effectuées à la jauge à écorce, sur chaque arbre d'une placette, ont permis de déduire les caractéristiques suivantes pour chaque placette : l'accroissement annuel en surface terrière sur écorce et l'accroissement annuel relatif correspondant. Cette dernière caractéristique a été calculée sous la forme $[\operatorname{LOG}(G)-\operatorname{LOG}(G+\Delta G)] / 5$ où LOG désigne le logarithme népérien et $\Delta G$ l'accroissement en surface terrière sur écorce correspondant à 5 ans; cette forme de calcul a l'avantage (voir FisHER, 1970 , p. 28) de ne pas surestimer ou sous-estimer l'accroissement relatif comme cela se passe lorsqu'il est obtenu en divisant laccroissement annuel par la valeur initiale ou la valeur finale de la surface terrière. Ces deux grandeurs furent successivement étudiées avec l'intention de rechercher une relation les liant à d'autres caractéristiques des peuplements, à savoir : la hauteur, l'âge et la surface terrière. L'analyse portant sur l'accroissement de la surface terrière et concernant une éventuelle relation entre cette variable et l'une des trois autres variables dernièrement citées fut négatif : les graphiques observés ne montraient qu'une dispersion cahotique des points. Par contre d'autres examens mettaient nettement en évidence une relation entre l'accroissement 
relatif en surface terrière et tour à tour la hauteur ou l'âge. C'est finalement entre l'accroisement relatif de la surface terrière et l'âge (A) qu'une relation était le plus clairement et le plus précisément définie (voir la figure 2). La relation estimée s'écrit :

$$
\frac{1}{G} \frac{d G}{d A}=12,8535 A^{-1,6}+0,0076
$$

La variabilité de l'accroissement relatif de la surface terrière non expliquée par l'âge n'a pu être ultérieurement réduite en faisant intervenir en plus de cette variable à nouveau la hauteur, la surface terrière, et de surcroît certaines mesures de la densité associées au nombre de tiges; la relation (2) a donc été considérée comme l'expression définitive de l'accroissement de la surface terrière. (Il est à noter que Décourt a pu utiliser avec succès l'accroissement relatif de la surface terrière pour l'étude de placettes permanentes : voir DécourT, 1971).

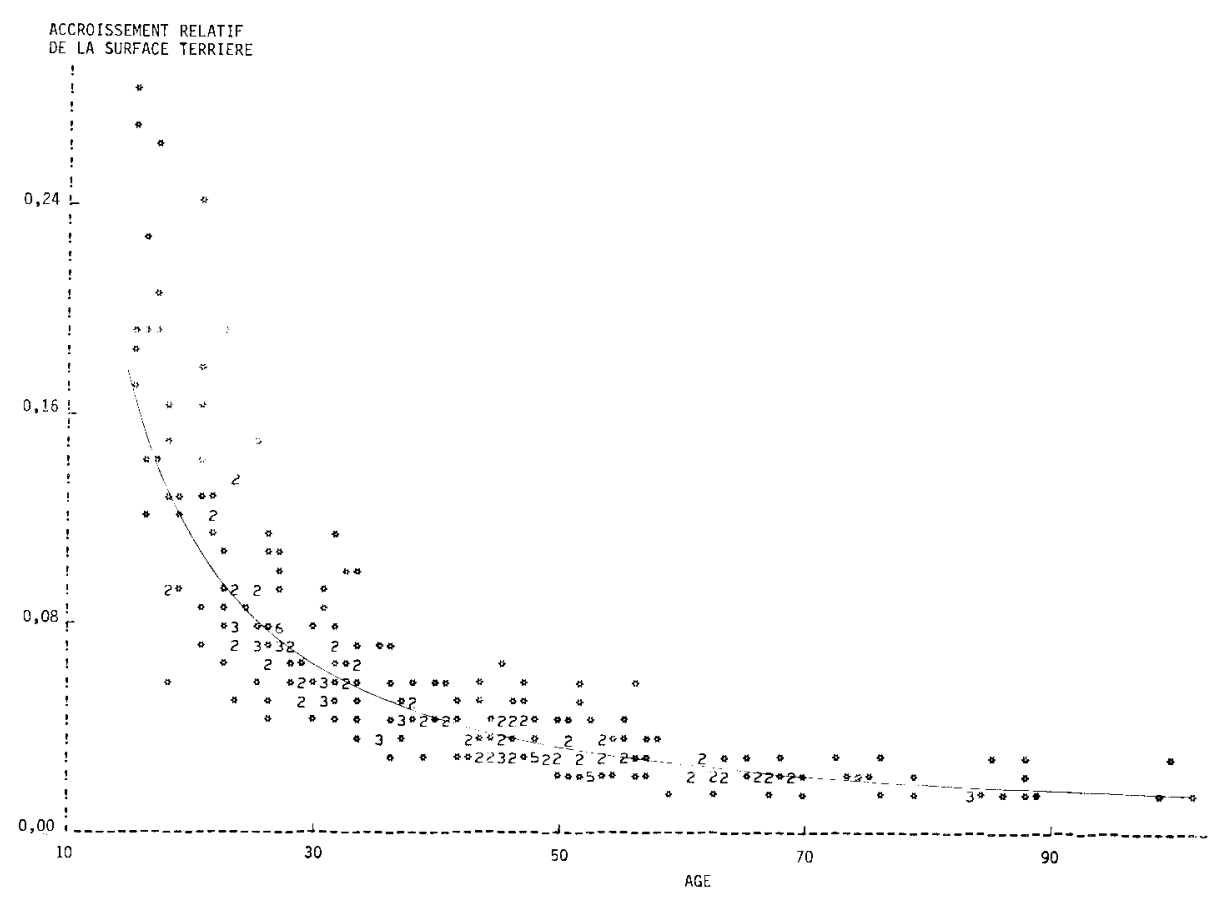

FIG. 2

Relation entre l'accroissement relatif de la surface terrière [i.e. $(d G / d A / G)]$ et l'âge des placettes

Relationship between relative basal area growth rate and age of the studied stands

Si l'on fait abstraction des causes de variabilité non contrôlées, le volume d'un peuplement donné est une fonction $\mathrm{V}$ du temps, sa surface terrière est une fonc- 
tion $\mathrm{G}$ du temps, et sa hauteur une fonction $\mathrm{H}$ du temps déterminée par la productivité de la station; ces grandeurs vérifient:

$$
\mathrm{V}=\mathrm{G} \mathrm{f}(\mathrm{H})
$$

où $f$ est la fonction définic par la relation (1). Pour un intervalle de temps suffisamment court pendant lequel des accidents ne viennent pas faire disparaître des arbres sur pied, la production totale VT a un accroissement égal à celui du volume sur pied (une démonstration plus formelle de ce fait est donnée par CurTIs, 1967); par conséquent, d'après (3) il vient :

$$
\frac{d V T}{d A}=\frac{d V}{d A}=\frac{d G}{d A} f(H)+G-\frac{d}{d A} f(H)
$$

ce qui peut encore s'écrire:

$$
\frac{d V T}{d A}=\left\{\frac{1}{G} \frac{d G}{d A} f(H)+\frac{d}{d A} f(H)\right\} G
$$

Dans cette équation différentielle qui détermine la production totale, tous les éléments peuvent être supposés connus excepté la fonction $G$ du temps qui représente l'évolution de la surface terrière du peuplement envisagé résultant des phénomènes de croissance et de mortalité. La question de la détermination de cette fonction nécessaire à l'estimation de la production totale est reportée à la section suivante.

\section{4. - Croissance et développement typiques des peuplements de pin sylvestre en Margeride}

La seule relation déterminant $G$ d'une façon qui puisse être exploitée pour intégrer l'équation (4) de la section précédente s'écrit:

$$
\mathrm{G}=15,3072 \mathrm{H}^{0, \overline{5}}-26,1899
$$

Cette relation qui s'est avérée être indépendante de lâge a été estimée dans les conditions suggérées par la figure 3 où il apparaît qu'elle se trouve vérifiée avec une faible précision. Dans ces conditions on peut s'interroger sur le sens qu'il y a de faire abstraction de la variabilité inexpliquée par la hauteur en utilisant la relation (5) pour décrire l'évolution de la surface terrière d'un peuplement. Or cette variabilité est due en grande partie aux différences de nombre de tiges entre les placettes d'âge et de productivités comparables (cela apparaîtra indirectement dans la prochaine section); ces différences proviennent elles-mêmes de la petite surface des placettes (variant approximativement de 1 à 7 ares) et du manque d'homogénéité des peuplements d'où elles sont issues. On peut donc raisonnablement supposer que pour un peuplement de plus grande surface (par exemple 1 ha) ces différences locales se compensent et que l'évolution de la surface terrière se réalise effectivement comme il est prévu par la relation (5). Quoi qu'il en soit cette relation a été utilisée pour permettre d'exprimer 
l'équation (4) en fonction du temps par lintermédiaire de courbes de croissance en hauteur dont la forme générale était issue de la première partie de l'étude. Trois hauteurs de référence à 50 ans : $15 \mathrm{~m}, 11,25 \mathrm{~m}, 7,50 \mathrm{~m}$ ont été retenues pour représenter l'intervalle des productivités de l'échantillon des placettes de l'étude, et les courbes de croissance en hauteur correspondantes caractérisent avec les relations (1), (2), (4), (5), la dynamique des peuplements de pin sylvestre en Margeride, illustrée par l'ensemble des courbes de la figure 4 (l'équation (4) a été intégrée numériquement).

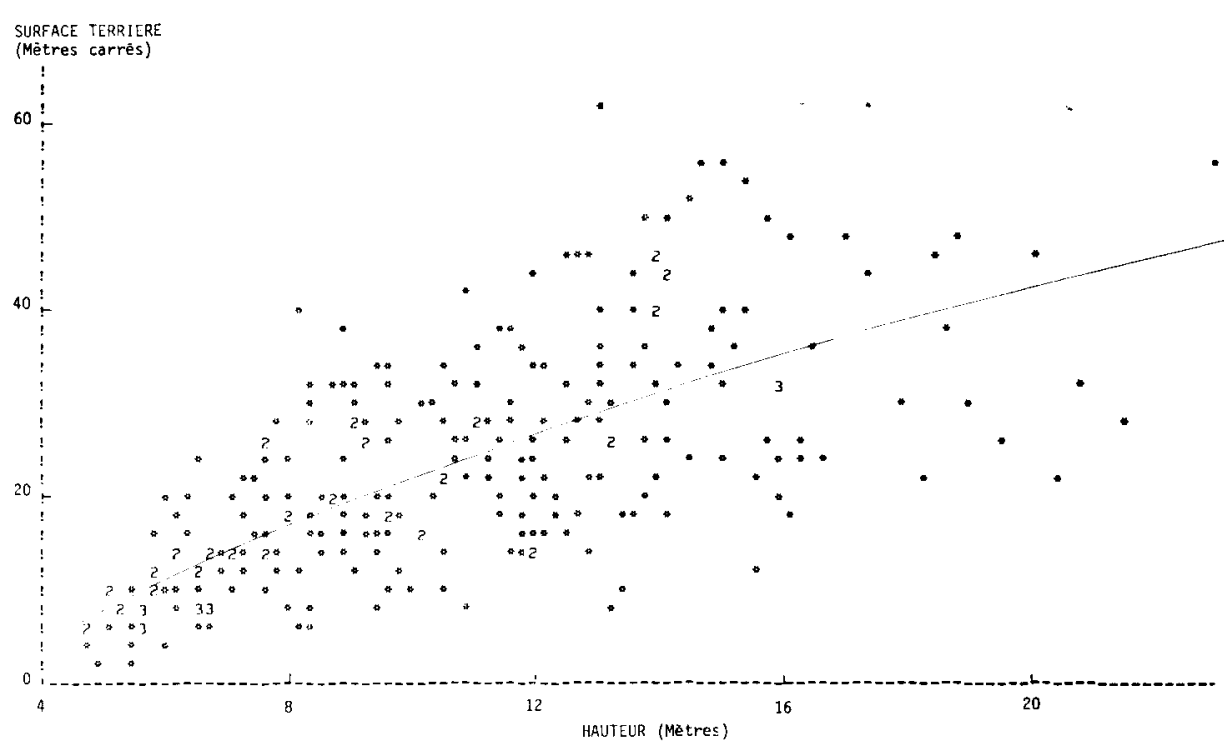

FIG. 3

Dispersion des points représentant les placettes de hauteur et de surface terrière données en abscisse et en ordonnée

Scatter diagram of hasal area versus height of the stands

A l'issue de cette section il est important de faire la remarque suivante : l'équation (4) laisse prévoir des variations de la production associées à des changements apportés à l'évolution du matériel sur pied (qui fixe la relation (5)); des variations plus sensibles que ne le fait généralement apparaître l'expérience (conformément à la loi de EichorN). Cela vient vraisemblablement du fait que laccroissement relatif de la surface terrière doit être assez longtemps sous l'influence des conditions de densité initiales qui n'ont pu être prises en compte lorsque la relation (2) a été établie (il apparaît en effet à la figure 2 que la variabilité de l'accroissement relatif de la surface terrière non expliquée par l'âge est plus importante pour les âges les plus bas). Pour ces raisons il serait hasardeux deffectuer un calcul de variation de la production totale avec la densité basé sur les relations estimées, car la relation (2) ne serait plus cohérente avec une loi d'évolution de la surface terrière autre que celle définie par la relation (5), et par conséquent ne présentant plus la même symétrie relativement 

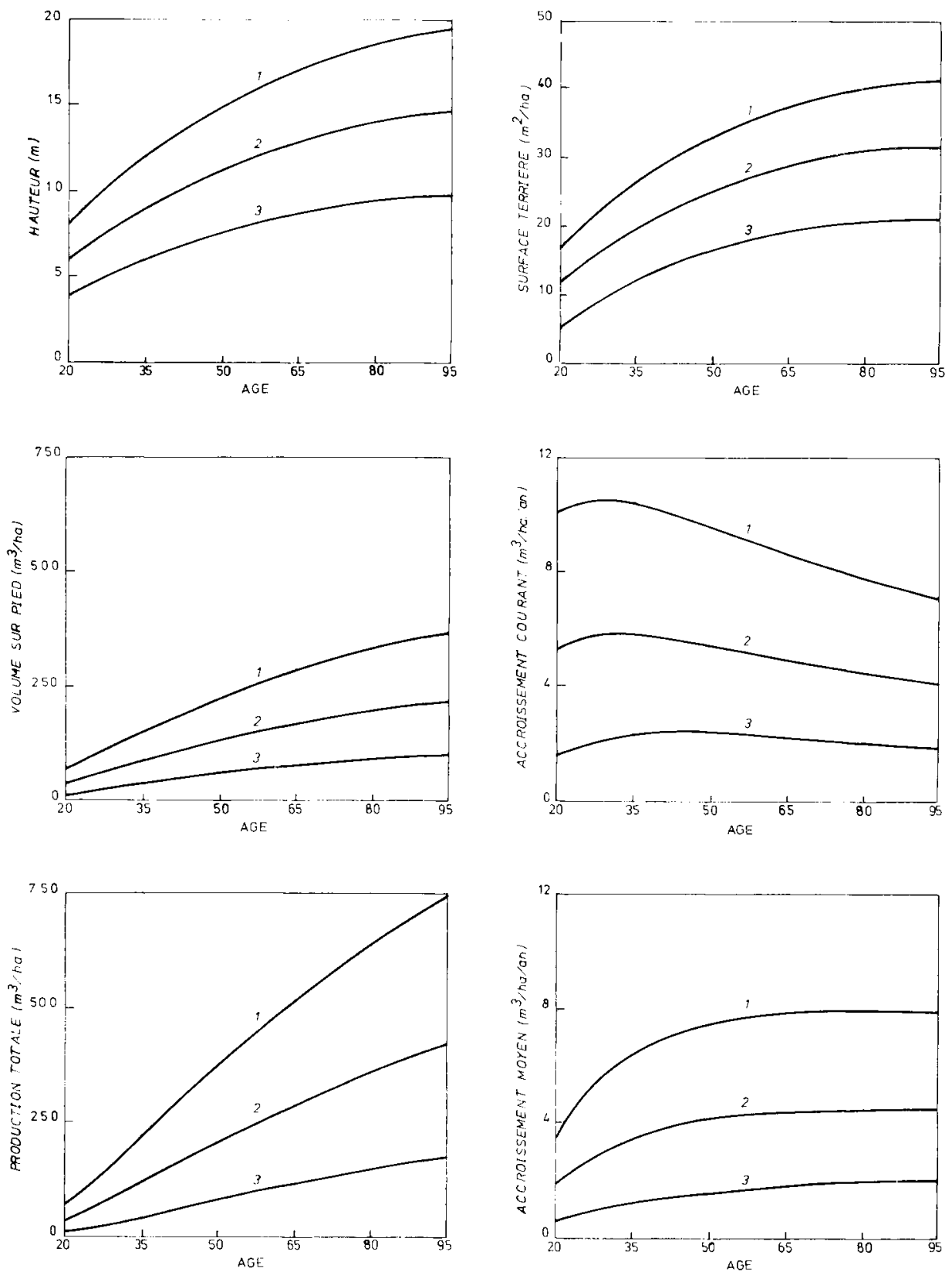

FIG. 4

Croissance et développement typiques des peuplement de pin sylvestre en Margeride pour trois classes de productivité

Typical growth and development of Scot pine stands in Margeride for three site classes 
aux observations. On peut cependant noter que si l'ordre de grandeur de ces variations prévu par la relation (4) n'est pas fiable, celles-ci ont lieu dans le sens généralement accepté : plus de matériel sur pied, donne une plus forte production totale (voir Möller, 1954, p. 31 ; BraAthe, 1957, p. 51).

\section{5. - Tables de production simplifiées pour le pin sylvestre en Margeride}

En vue d'une utilisation plus pratique des résultats de la section précédente, il est déjà possible, pour les classes de productivité représentées par les hauteurs de référence choisies, de tabuler (par exemple par décade) les caractéristiques de peuplements suivantes : l'âge, la hauteur, la surface terrière, le volume, l'accroissement courant, la production totale, laccroissement moyen. On peut donc obtenir ainsi des tables de production simplifiées représentant la croissance et le développement des peuplements comme cela se fait habituellement. Il resterait à faire figurer dans cette table le nombre de tiges, ce qui est le premier objet de cette section.

\section{1. - Evolution du nombre de tiges}

L'analyse des données a permis d'établir que le nombre de tiges à l'unité de surface était lié simultanément aux caractéristiques de peuplement suivantes : la surface terrière, la hauteur et l'âge. Cette analyse n'a pu procurer aucun résultat réellement positif avant qu'il n'ait été appliqué une transformation logarithmique au nombre de tiges, car l'irrégularité et la forme complexe de dépendance de cette variable relativement aux autres caractéristiques de peuplement citées ne permettait pas de mettre leur rôle en évidence. Une relation déterminant le logarithme du nombre de tiges en fonction de la surface terrière, de la hauteur, et de lâge, pourrait donc être donnée. Il est préférable cependant d'en donner une autre expression équivalente, à la fois plus aisément interprétable et plus conforme à l'application qui en sera faite. Pour l'écrire il est nécessaire de distinguer d'une part la valeur observée de la surface terrière $G$ et d'autre part sa valeur estimée pour une hauteur de peuplement donnée $\mathbf{H}$ par la relation (5) et notée GEST. Dans ces conditions le nombre de tiges $\mathrm{N}$ est déterminé par la relation:

$$
\operatorname{LOG}(\mathrm{N})=6,0491+3,0414(\mathrm{G} / \mathrm{GEST})^{0.5}+0,0151 \text { GEST }-0,7594 \operatorname{LOG}(\mathrm{A})
$$

ou encore, en faisant apparaître les valeurs résiduelles GRES $=\mathrm{G}$ - GEST :

$$
\begin{gathered}
\operatorname{LOG}(\mathrm{N})=6,0491+3,0414 \begin{array}{r}
1+ \\
+ \\
\quad+0,0151 \text { GEST }-0,7594 \operatorname{LOG}(\mathrm{A})
\end{array} \\
\mathrm{R}^{2}=0,77 \quad(268 \text { observations })
\end{gathered}
$$

( $\mathrm{N}$ en nombre de tiges par hectare)

Le second terme de la relation (6) est constant pour les valeurs de la surface terrière situées sur la trajectoire définie par la relation (5) (celles donc qui apparaîtront dans les tables de production proposées); ce terme en effet rend seulement compte de la 
variation du nombre de tiges associée aux écarts à cette trajectoire. Par ailleurs en l'absence de ces écarts les deux termes suivants rendent compte de l'évolution du nombre de tiges suivant l'âge et la productivité. Il est important de noter que les deux derniers termes de la relation (6) où apparaissent dans l'un la hauteur et dans l'autre âge (sous certaines formes) ne sont pas redondants; au contraire, ils tendent à traduire le fait que le nombre de tiges de peuplements ayant même hauteur décroît avec la productivité des stations. Afin d'apprécier le degré de certitude de ce fait, et comme les conditions de l'estimation le permettaient, des tests statistiques, basés sur l'hypothèse de normalité ont été effectués et ont confirmé que chacun des paramètres estimés en question šécartait très significativement de zéro eu égard à la variabilité inexpliquée.

\section{2. - Tables de production}

La relation (6) permet done de compléter les indications possibles des tables de production décrites au début de cette section. Dans ces tables qui constituent le tableau 1 les valeurs estimées du nombre de tiges n'ont pas été obtenues par simple exponentiation de la relation (6). En effet, les valeurs du logarithme du nombre de tiges observé se dispersaient assez régulièrement pour être supposées normales comme cela a déjà été signalé, et dans ces conditions une estimation sans biais du nombre de tiges moyen correspondant à une hauteur et à un âge donnés a pu être obtenue grâce à un important résultat de FinNey (1941) adapté par Bradu \& MUNDLAK (1970), qui a été mis en application pour la circonstance (d'après l'article cité de FinNeY, si $Y=\operatorname{LOG}(\mathrm{X})$ suit une loi normale, les estimateurs bien connus de la variance et de la moyenne de $\mathrm{Y}$ issus de la méthode des moindres carrés sont indépendants ; cette propriété est fortement utilisée pour déterminer le biais de $\operatorname{EXP}(\bar{Y})$ pris comme estimateur de la moyenne de $\mathrm{X}$ ).

Il n'a pas été procédé à une véritable épreuve de validité des tables proposées, mais certaines comparaisons ont pu être faites avec quelques données provenant de placettes permanentes de pin sylvestre étudiées par Marquestaut (1979) dans la même région. Pour 9 de ces placettes permanentes qui se trouvent dans des stations de bonne productivité, les accroissements courants ont été publiés; ils se situent entre les valeurs indiquées aux âges correspondants (de 48 à 68 ans) par les tables des classes 1 et 2 du tableau 1 ; il en est de même pour les volumes sur pied qui se trouvent de la même façon encadrés par les valeurs du tableaul 1. Par contre les nombres de tiges de ces placettes permanentes sont nettement plus élevés que ceux prévus par le tableau 1 dans les conditions correspondantes. BARBET (1978) aussi dans son étude du pin sylvestre en Margeride a donné des chiffres beaucoup plus élevés pour les nombres de tiges. Ce désaccord peut être dû en partie au fait que les tiges de moins de $7,5 \mathrm{~cm}$ de diamètre ne sont pas recensées par l'I.F.N., mais il vient aussi très probablement de ce que l'on a tendance à installer les placettes de production dans des peuplements fermés et présentant un aspect de futaie régulière. Au contraire de cela, les placettes d'un inventaire proviennent d'un échantillonnage aléatoire et ¿doivent représenter toutes les conditions de peuplement. Par ailleurs, d'après un contrôle qui a été effectué a posteriori avec les données de cette étude, il semble bien que les circonférences moyennes de peuplement prévues par le tableau - c'està-dire déduites des tables de production par le calcul portant sur le nombre de tiges et la surface terrière qu'elles indiquent — soient compatibles avec celles observées sur les placettes échantillon ayant servi à établir les tables. 


\section{TABLEAU 1}

Tables de production simplifiées pour les peuplements de pin sylvestre en Margeride.

Les productions moyennes maximum pour les trois classes de fertilité se situent respectivement vers 78 ans, 83 ans et 90 ans

pour les trois classes de productivité représentées de la $1^{\text {re }}$ à la $3^{\circ}$

(les valeurs sont à l'ha, hauteurs en $m$, surfaces terrières en $m^{2}$, volumes en $m^{3}$ )

Yield tables for Scot pine in Margeride

\begin{tabular}{|c|c|c|c|c|c|c|c|}
\hline AIIE & HAL_TEELIال_K & $\begin{array}{l}\text { NDMEFE } \\
\text { LEE } \\
\text { TILES }\end{array}$ & 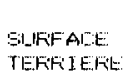 & $\begin{array}{c}\text { VILIINIE } \\
\text { SI_IF } \\
\text { FELI }\end{array}$ & $\begin{array}{l}\text { ALLLFICII SEEMENT } \\
\text { GLLHISANT }\end{array}$ & 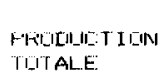 & $\begin{array}{l}\text { ALIEFII ESEMENI } \\
\text { WIIYEN }\end{array}$ \\
\hline$=0$ & $\Xi .1$ & 12,0 & 17 & $6 \%$ & 10.1 & 69 & 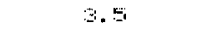 \\
\hline 30 & 10.9 & $10 \%$ & 24 & 12 & 10,5 & $1 \%$ & $=1$. \\
\hline 40 & 13.2 & : & 2 & $17 \%$ & 10.2 & $\because 77$ & 6.9 \\
\hline 50 & 15.0 & $E x 1$ & 3 & 227 & 9 & : & 7.5 \\
\hline$\Leftrightarrow$ & 16.4 & $7=7$ & 6 & $\because \Leftrightarrow$ & 9.0 & 470 & $7.8:$ \\
\hline 70 & 17.8 & 6.7 & $=$ & $=4$ & $\because .4$ & 557 & $\because .0$ \\
\hline$\$ 0$ & 13.5 & 613 & 40 & 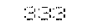 & 7.5 & $\therefore=4$ & $\because 0$ \\
\hline 90 & $1 \% 2$ & $5 \%$ & 4.1 & 97 & 7.4 & 714 & 7.9 \\
\hline
\end{tabular}

ILAOE 2

\begin{tabular}{|c|c|c|c|c|c|c|c|}
\hline$A C B=$ & HAIITIEUIHE & $\begin{array}{c}\text { NLMEFE: } \\
\text { LEE } \\
\text { TILESE }\end{array}$ & 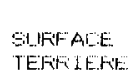 & 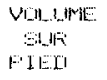 & 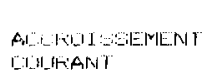 & 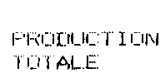 & 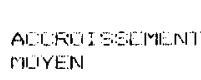 \\
\hline $\mid 20\}$ & G. 1 & $11 \leqslant$ & 12 & 37 & 5. & $: 3$ & $1 . \%$ \\
\hline 30 & E. & 9 & $1: 3$ & 71 & 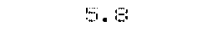 & 54 & 3.1 \\
\hline 40 & 9 & $6=$ & $2 z$ & 164 & 5. & 152 & 5. \\
\hline 50 & 11.2 & 710 & 2 & $1=2$ & $=\ldots$ & 20 & 4. $\cong$ \\
\hline 0 & 12. & 5.41 & 20 & $1: 97$ & $\because 2$ & $2=$ & 4.1 \\
\hline 70 & 13.2 & $=6:=i 6$. & $2 \%$ & $17 \%$ & 4.5 & 312 & 4.5 \\
\hline$\because 0$ & 13.6 & $\therefore 41$ & 31 & $1 \%$ & 4.6 & $\because k$ & 4. \\
\hline 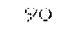 & 14.4 & 50 & 2 & 210 & 4..$=$ & 40 & 4.5 \\
\hline
\end{tabular}

LLAGEE Z

\begin{tabular}{|c|c|c|c|c|c|c|c|}
\hline AlBE & HALITEEIIFi & $\begin{array}{l}\text { NOINLAFIE: } \\
\text { IIE } \\
\text { T IEIE: }\end{array}$ & $\begin{array}{l}\text { SI_HIFAl.E } \\
\text { TEFIFIEFEE }\end{array}$ & $\begin{array}{l}\text { WILILIVIE } \\
\text { G_LF } \\
\text { FIE[I }\end{array}$ & $\begin{array}{l}\text { ALILFILI SEENHENI } \\
\text { CILIFIANIT }\end{array}$ & 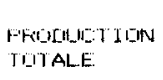 & $\begin{array}{l}\text { AL_LFEI I SEEMIENV I } \\
\text { MH_YYEN }\end{array}$ \\
\hline 1201 & 4.0 & 1042 & 5 & $1 z$ & 1.6 & $1:=$ & 0.0 \\
\hline$(:=10)$ & 5.5 & $: 27$ & 10 & 2 & : & 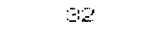 & 1.1 \\
\hline 40 & $E . E$ & 701 & 13 & 4 & 2.4 & 5 & 1.4 \\
\hline 50 & 7.5 & $\therefore 10$ & 15 & 5 & 2.4. & $7 \%$ & 1.5 \\
\hline$\therefore 0$ & 5.2 & $x+2$ & 13 & 71 & 28 & 100 & 1.7 \\
\hline 70 & 5.8 & $\therefore \mathrm{Cr}_{2}$ & $1 \%$ & $\because 2$ & $\therefore$ & 10 & 1.: \\
\hline 513 & 9.2 & $4 \Leftrightarrow 2$ & 20 & 0 & $\therefore 1$ & 145 & 1.5 \\
\hline 80 & 9.0 & $4=3$ & 21 & 77 & 1.9 & $1 \Leftrightarrow$ & $1 . E$ \\
\hline
\end{tabular}

En effet, une relation liant la circonférence moyenne des peuplements $C G$ à leur hauteur et à leur âge a pu être établie directement à partir des circonférences moyennes observées, malgré une variabilité résiduelle assez importante.

$$
\begin{gathered}
\mathrm{CG}=-0,47663+0,02075 \mathrm{H}+0,23782 \operatorname{LOG}(\mathrm{A}) \\
\mathrm{R}^{2}=0,66(268 \text { observations })
\end{gathered}
$$




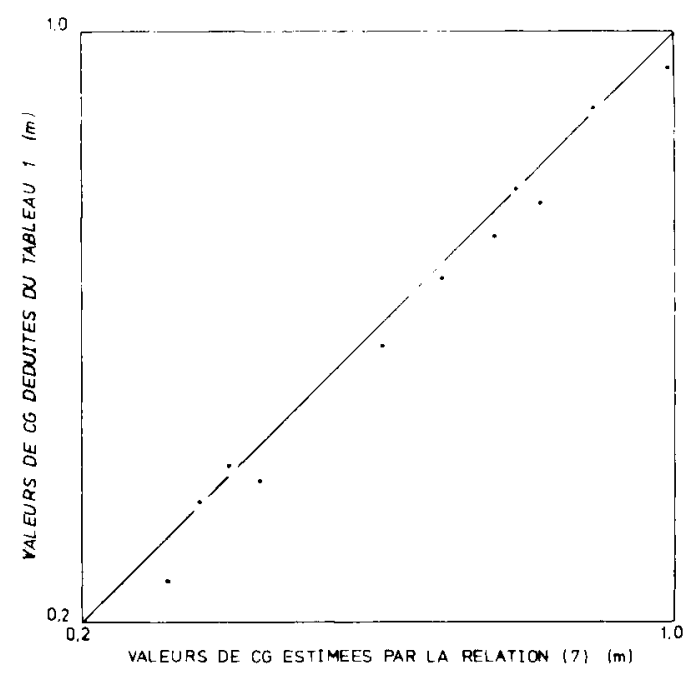

FIG. 5

Comparaison de deux estimations de la circonférence movenne des placettes de caractéristiques typiques pour différentes valeurs de l'âge et de la hauteur

Comparison of two different sets of estimated values of the mean girth at breast height for typical stands at different age and heights: calculated values from table 1 against estimation directly obtained from the observed data in table I with relation (7)

La figure 5 permet de comparer les circonférences moyennes estimées par cette relation pour diverses valeurs de $\mathrm{H}$ et de $\mathrm{A}$ lues au tableau 1, avec les valeurs de la circonférence moyenne calculées à partir de la surface terrière et du nombre de tiges correspondants lus dans le même tableau : la différence entre deux valeurs obtenues pour la circonférence moyenne d'un peuplement de hauteur et d'âge donnés est nulle lorsque le point représentatif ayant l'une pour abscisse et l'autre pour ordonnée se trouve sur la première bissectrice du repère. La comparaison entre les deux ensembles de valeurs est assez satisfaisante : compte tenu de la différence de nature entre les valeurs comparées qui introduit nécessairement un biais (les unes proviennent d'une estimation directe, les autres d'un calcul sur des estimations), la figure 5 ne permet pas de déceler un grave défaut systématique des tables de production proposées au tableau 1, du point de vue de l'évolution du nombre de tiges associce à une évolution donnée de la surface terrière; il est cependant prudent de considérer comme des extrapolations la première ligne de la deuxième table : peuplements de la 2 " classe de productivité à 20 ans, et les deux premières lignes de la dernière table : peuple-

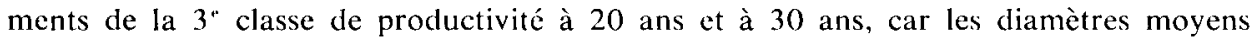
correspondants sont trop proches du seuil de $7,5 \mathrm{~cm}$.

En application de cette dernière analyse, la figure 6 montre l'évolution de la circonférence moyenne des peuplements avec la hauteur pour les trois classes de productivité représentées par les tables, et permet de comparer de ce point de vuc les peuplements de pin sylvestre en Margeride avec ceux de Sologne étudiés par Dé- 


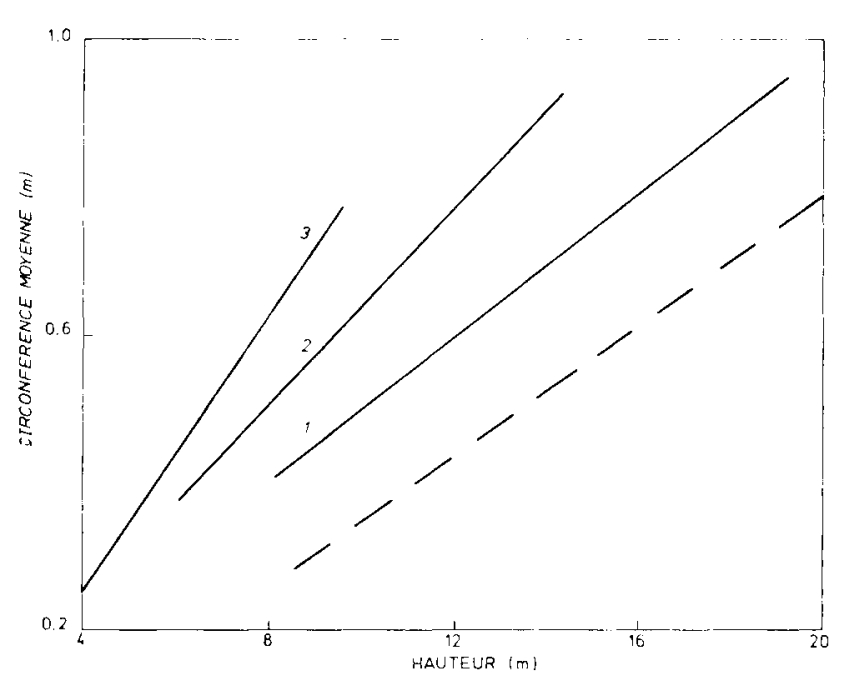

FIG. 6

Evolution de la circonférence moyenne avec la hauteur pour les trois classes de productivité représentées à la figure 4;

les tirets donnent cette évolution pour le pin sylvestre en Sologne (Décourt, 1965)

Variation of girth at breast height with height for the three site classes featuring in figure 4. The dashed line allows for comparison of this variation for Scot pine in a different region where - as opposed to Margeride - it is mostly planted

COURT (1965). Le fait que pour les peuplements de Sologne le nombre de tiges à l'hectare correspondant à une hauteur donnée est plus important apparaît indirectement sur cette figure. Malgré la définition particulière qui fut adoptée pour la hauteur de peuplement $\mathrm{H}$ : la hauteur moyenne des arbres dominants et codominants, cette comparaison est possible; il s'est en effet avéré que cette hauteur - elle devait procurer une caractéristique moins sensible dans certains cas à la présence d'arbres de trop petites dimensions relatives et ne participant plus à la dynamique du peuplement pour la grande majorité des placettes coïncidait avec la hauteur moyenne. Cette précision offre l'occasion de signaler qu'en pratique, pour l'utilisation du tableau 1, il n'y a pas d’inconvénient à confondre la hauteur indiquée avec la hauteur moyenne.

\section{6. - Conclusion}

Il est très remarquable que les données initialement destinées à un inventaire forestier sur une grande échelle aient pu être exploitées dans le cadre d'une étude de production pour permettre de parvenir à des indications sur les caractéristiques essentielles de la croissance et du développement d'une essence dans une région. Il est encore difficile de pouvoir vraiment juger ces résultats qui semblent malgré tout 
acceptables; cependant des chiffres sur la production totale ont été obtenus et des traits particuliers aux peuplements de pin sylvestre en Margeride ont pu être mis en évidence. D'après les dernières phases de l'étude, le type des peuplements représenté par le tableau 1 faisant la synthèse des données utilisées paraît être celui de peuplements parfois un peu clairsemés que lon rencontre en Margeride (comme les bois de ferme) plutôt que le reflet de futaies régulières très denses. Il est très probable qu'en partant de la même source de données il soit possible pour d'autres essences forestières d'obtenir des renseignements de base sur leur production en procédant de façon similaire.

Reçu pour publication en décembre 1980.

\section{Remerciements}

1) La collaboration de M. J. Bertrand, chef de l'Echelon de Nancy de l'Inventaire Forestier National, a rendu accessibles les données qui ont permis d'effectuer les travaux présentés sous le titre "Application des données de l'I.F.N. à l'étude du pin sylvestre en Margeride».

2) Au cours de ces travaux, large usage a été fait de la programmathèque de la Station de Biométrie du C.N.R.F. installée sur I'IRIS 80 (sous le système d'exploitation SIRIS 8, C 10) de l'Institut Universitaire de Calcul Automatique de Lorraine.

3) D'autre part, une aide du personnel technique de cette station et en particulier celle de $M^{\text {"le }}$ E. VACHEZ a permis de réaliser une application basée sur l'ouvrage de D. MC NEIL déjà cité, permettant grâce au système de temps partagé de l'ordinateur utilisé de procéder rapidement (sur le mode conversationnel) à de très nombreuses analyses graphiques suivant les principes exposés dans la première section de cet article.

\section{Summary}

Study of growth and yield of Scot pine in Margeride using data from I.F.N.

$$
B \text { - The gross volume growth }
$$

In the second part of a study dedicated to the estimation of the gross volume yield of Scot pine in Margeride (an agricultural region of France), the relationships between the characteristics of studied stands were investigated to obtain an expression of the volume of the stands and of their current volume increment that could meet the initial needs. This led to a relation connecting the current volume increment to age, height, and basal area. Using height curves previously obtained in the first part of the study, and a relation expressing stand basal area as a function of height, the expression of current volume increment could have been integrated with respect to age for given site classes to have the corresponding gross volume yield. Adding to the knowledge of the dynamics of the stands, typical number of stems per hectare corresponding to given age, height and basal area could have been obtained. 


\section{Références bibliographiques}

Barbet C., 1978. Le pin sylvestre en Margeride. Mémoire de $3^{\circ}$ année E.N.I.T.E.F., 45290 Nogent-sur-Vernisson.

BRAathe P., 1957. Thinnings in even-aged stands, a summary of European literature. Fac. For. Univ. N. Brunsw., 91 p.

Pradu D., Mundlak Y., 1970. Estimation in lognormal linear models. J. Am. Stat. Assoc., 65 (329), 198-211.

Buckman R., 1962. Growth and yield of red pine in Minnesota. U.S. Dept. Agric. Tech. Bull,, $1972,50 \mathrm{p}$.

Clutter J., 1963. Compatible growth and yield models for loblolly pine. For. Sci., 9 (3), 354-371.

CurTis R.O., 1967. A method of estimation of gross yield of Douglas-fir. For. Sci. Monograph., 13, 24 p.

Décourt N., 1965. Le pin sylvestre et le pin laricio de Corse en Sologne. Ann. Sci. for., $22(2), 259-317$.

DécourT N., 1971. Remarque sur l'allure de la croissance en surface terrière dans le carré latin de douglas d'Amance. Document non publié rédigé à la Station de Sylviculture et de Production du C.N.R.F., 6 p., 3 fgs.

DÉCOURT N., 1972. Méthode utilisée pour la construction rapide de tables de production provisoires en France. Ann. Sci. for., 29 (1), 35-48.

Draper N.R., Smith H., 1966. Applied regression analysis. John Wiley and Sons, New York.

FISHER R.A., 1970. Statistical methods for research workers. Oliver and Boyd, Edinburgh, 14th edition.

FinNey D.J., 1941. On the distribution of a variate whose logarithm is normally distributed Supplement to the J. Royal Statist. Soc., 7, 151-161.

MC NeIL D., 1977. Interactive Data Analysis. John Wiley and Sons, New York.

Marquestaut J., 1978. Quelques données sur les peuplements de pin sylvestre en Margeride. A.F.O.C.E.L., Ann. Rech. Sylvic., 2, 318-351.

MöLLER C.M., 1954. The influence of thinning on volume increment. In thinning problems and practices in Denmark. N.Y. State Coll. For. Tech. Publ., 76, 5-44

Mosteller F., Tukey J.W., 1977. Data analysis and regression. Addison-Wesley, Reading (Mass.).

Otrorini J.-M., NYs C., 1981. Application des données de l'I.F.N. à l'étude de la production du pin sylvestre en Margeride. A. - Etude de la croissance en hauteur. Ann. Sci. for., 38 (2). 